Check for updates

Cite this: RSC Adv., 2018, 8, 29589

\title{
Ingenious modification of molecular structure effectively regulates excited-state intramolecular proton and charge transfer: a theoretical study based on 3-hydroxyflavone
}

\begin{abstract}
Jianhui Han, (D) Xiaochun Liu, Chaofan Sun, You Li, Hang Yin and Ying Shi (DD*
Harnessing ingenious modification of molecular structure to regulate excited-state intramolecular proton transfer (ESIPT) and intramolecular charge transfer (ICT) characteristics holds great promise in fluorescence sensing and imaging. Based on the 3-hydroxyflavone (3HF) molecule, 2-(2-benzo[b] furanyl)-3-hydroxychromone (3HB) and 2-(6-diethylamino-benzo[b]furan-2-yl)-3-hydroxychromone (3HBN) were designed by the extension of the furan heterocycle and the introduction of a diethylamino group. The analysis of important hydrogen bond length, frontier molecular orbitals, infrared spectra, and potential curves have cross-validated our results. The results indicate that proper site furan heterocycle extension and diethylamino donor group substitution not only shift the absorption and emission spectra to the red but also effectively modulate the excited-state dynamic behaviors. Strengthened ICT characteristics from $3 \mathrm{HF}$ to $3 \mathrm{HB}$ and to $3 \mathrm{HBN}$ make the occurrence of ESIPT increasingly difficult due to the higher energy barriers, which indicates that the ESIPT and ICT processes are competitive mechanisms. We envision that our work would open new windows for improving molecular properties and developing more fluorescent probes and organic radiation scintillators.
\end{abstract}

Received 8th July 2018
Accepted 9th August 2018

DOI: $10.1039 / c 8 r a 05812 a$

rsc.li/rsc-advances

\section{Introduction}

Flavones and related compounds of the flavonoid group are widespread in plants ${ }^{1}$ and their importance is not limited to the survival of plants in the natural environment. They can effectively protect photo-sensitive biological targets such as DNA, proteins, polymers and phospholipids. ${ }^{2-5}$ More importantly, flavone compounds have a broad range of therapeutic applications such as reducing agents, hydrogen-donating antioxidants, active against cancers, tumors and cardiac problems and having low systemic toxicity. ${ }^{6-10}$ These extensive applications are closely related to their intrinsic physical characteristics. A large number of studies regarding excited-state intramolecular proton transfer (ESIPT) and intramolecular charge transfer (ICT) processes have been reported in different systems. ${ }^{\mathbf{1 1 - 1 4}}$ As a representative member of the flavones family, 3-hydroxyflavone (3HF) is well known for its characteristics of ESIPT and ICT processes. ${ }^{15-19}$ However, the absorption maximum in the near UV region and narrow fluorescence emission wavelength scope for $3 \mathrm{HF}$ greatly restrict its applications as probes in molecular and cellular biology and in nanoscale sensor technologies. Therefore, it is urgent to look for effective methods to

Institute of Atomic and Molecular Physics, Jilin University, Changchun 130012, China. E-mail: shi_ying@jlu.edu.cn; Fax: +86-431-85168816; Tel: +86-431-85168817 improve these properties. Fortunately, Chou and his colleagues in previous studies have demonstrated that the addition of $4^{\prime}$ dialkylamino group on the $\beta$-phenyl ring of $3 \mathrm{HF}$ can greatly

Table 1 Calculated absorption peak values $(\mathrm{nm})$ with different functional methods and experimental (Exp.) data in toluene solvent for $3 \mathrm{HF}$, $3 \mathrm{HB}$, and $3 \mathrm{HBN}$ structures

\begin{tabular}{llllll}
\hline & M062X & CAM-B3LYP & B3LYP & PBE0 & Exp. $^{a}$ \\
\hline 3HF & 313 & 318 & 354 & 343 & 343 \\
3HB & 335 & 344 & 392 & 378 & 364 \\
3HBN & 392 & 381 & 478 & 455 & 446
\end{tabular}

${ }^{a}$ See ref. 24.

Table 2 Calculated the oscillator strengths $(f)$ in first two excited-state and corresponding frontier molecular orbital transitions (MOs) of $3 \mathrm{HF}$, $3 \mathrm{HB}$, and $3 \mathrm{HBN}$ structures are listed

\begin{tabular}{llll}
\hline & Transition & $f$ & MOs \\
\hline $3 \mathrm{HF}$ & $\mathrm{S}_{0} \rightarrow \mathrm{S}_{1}$ & 0.54 & H-L \\
& $\mathrm{S}_{0} \rightarrow \mathrm{S}_{2}$ & 0.00 & H-4-L \\
$3 \mathrm{HB}$ & $\mathrm{S}_{0} \rightarrow \mathrm{S}_{1}$ & 0.83 & H-L \\
& $\mathrm{S}_{0} \rightarrow \mathrm{S}_{2}$ & 0.03 & H-1-L \\
$3 \mathrm{HBN}$ & $\mathrm{S}_{0} \rightarrow \mathrm{S}_{1}$ & 0.89 & H-L \\
& $\mathrm{S}_{0} \rightarrow \mathrm{S}_{2}$ & 0.08 & H-1-L
\end{tabular}



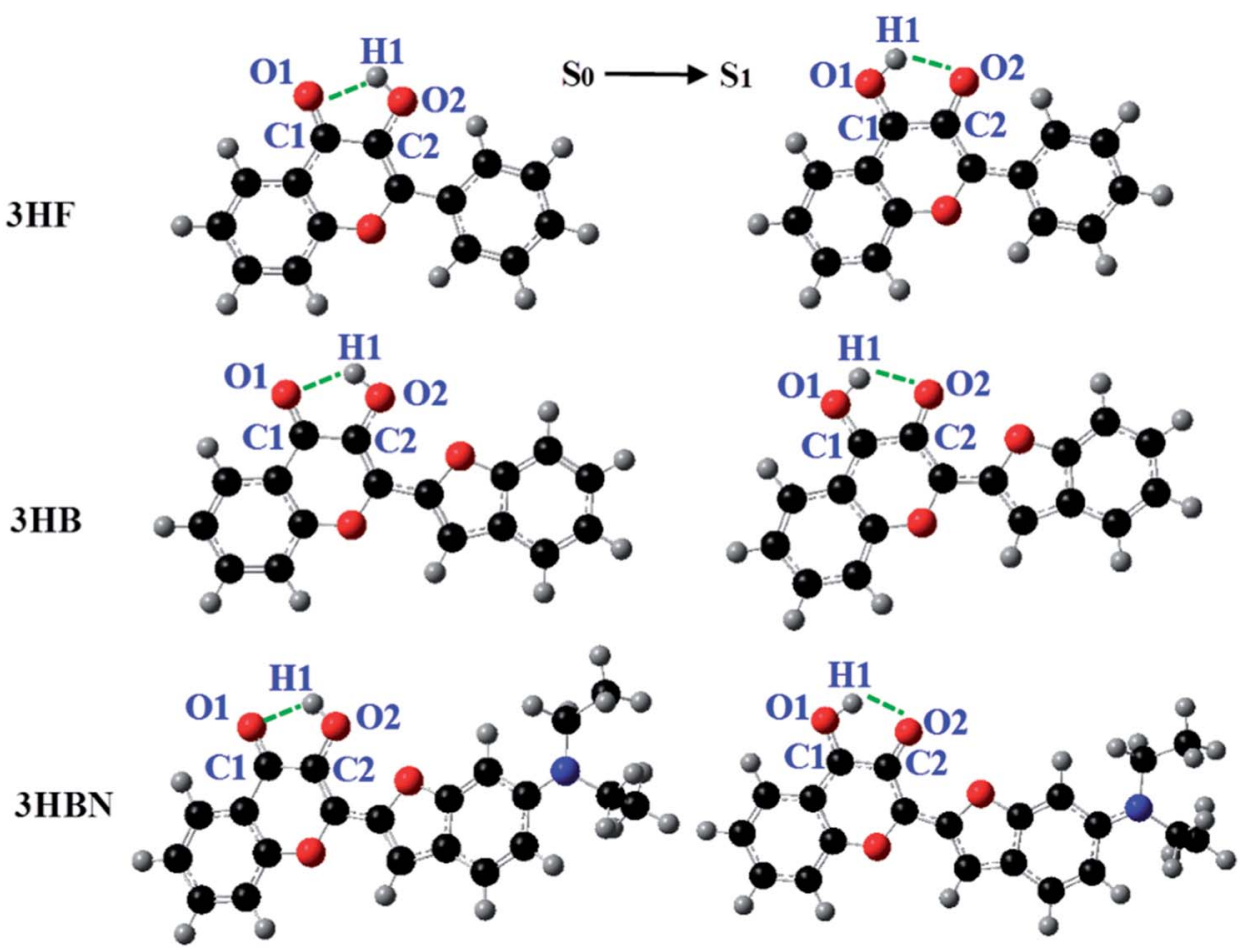

Fig. 1 Optimized geometric structures of $3 \mathrm{HF}, 3 \mathrm{HB}$ and $3 \mathrm{HBN}$ in the ground-state and excited-state.

change the electron density distribution and affects ICT dynamic process. $^{20,21}$ Klymchenko et al. have synthesized experimentally the structures of 2-(2-benzo[b]furanyl)-3hydroxychromone (3HB) and 2-(6-diethylamino-benzo $[b]$ furan2-yl)-3-hydroxychromone (3HBN) based on the simple $3 \mathrm{HF}$ structure. ${ }^{22-25}$ And they have elaborated that the introduction of furan heterocycle on 3HF derivatives showed stronger fluorescence emission and longer emission position. However, the underlying causes for the effect of changing functional groups on ICT dynamic process, and the internal connection between ESIPT and ICT processes of $3 \mathrm{HF}$ remain unclear.

In our work, we substituted 2-(2-benzo[b]furanyl) for 2phenyl and introduced diethylamino group to form $3 \mathrm{HB}$ and $3 \mathrm{HBN}$ structures separately based on original $3 \mathrm{HF}$ structure. The attention is focused on analyzing the regulation of absorption and emission spectra by structural changes and exploring the relationship between ESIPT and ICT dynamic processes of $3 \mathrm{HF}, 3 \mathrm{HB}$ and $3 \mathrm{HBN}$ molecules. Achieving absorption and fluorescence spectra shift toward the desired direction and understanding the dynamic relationship between ESIPT and ICT processes are valuable for the future molecular design and the modulation of dynamic progress.

\section{Computational methods}

All the calculations presented were accomplished depending on the Gaussian 09 program suite. ${ }^{26}$ The hybrid functional PBE0 was performed in current system and it was composed of $25 \%$ Hartree-Fock exchange and $75 \% \mathrm{PBE}^{27-30}$ In fact, a number of functionals, including B3LYP, PBE0, CAM-B3LYP, M062X were tested before designating functional, among which the PBE0 functional provides the most satisfactory agreement with experimental results. The triple- $\zeta$ valence quality with one set of polarization functions (TZVP) was used as basis sets. ${ }^{31}$ The geometric configurations of the $3 \mathrm{HF}, 3 \mathrm{HB}$, and $3 \mathrm{HBN}$ were optimized using the density functional theory (DFT) method in the ground-state and the time-dependent DFT (TDDFT) method in the excited-state, respectively. ${ }^{32-37}$ The TDDFT method has become a very useful tool to analyze the hydrogen bonding in the excited-states of the hydrogen-bond system theoretically. ${ }^{38-41}$ In the optimized structures, vibrational frequencies were analyzed to obtain thermodynamic corrections and to confirm the structures were minima (no imaginary frequency). Considering that the experiments were implemented in toluene solvent, the solvent corrections were also taken into account in all computations. The integral equation formalism variant of polarizable continuum model (IEFPCM) with radii and cavity-

Table 3 Calculated important bond lengths $(\AA)$ for the optimized structure of $3 \mathrm{HF}, 3 \mathrm{HB}$ and $3 \mathrm{HBN}$ in the ground-state $\left(\mathrm{S}_{0}\right)$ and in the excited-state $\left(\mathrm{S}_{1}\right.$ and $\left.\mathrm{S}_{1}^{\prime}\right)$

\begin{tabular}{|c|c|c|c|c|c|c|c|c|c|}
\hline & \multicolumn{3}{|l|}{$3 \mathrm{HF}$} & \multicolumn{3}{|l|}{$3 \mathrm{HB}$} & \multicolumn{3}{|l|}{$3 \mathrm{HBN}$} \\
\hline & $\mathrm{S}_{0}$ & $\mathrm{~S}_{1}$ & $\mathrm{~S}_{1}^{\prime}$ & $\mathrm{S}_{0}$ & $\mathrm{~S}_{1}$ & $\mathrm{~S}_{1}^{\prime}$ & $\mathrm{S}_{0}$ & $\mathrm{~S}_{1}$ & $\mathrm{~S}_{1}^{\prime}$ \\
\hline $\mathrm{H}_{1}-\mathrm{O}_{2}$ & 0.979 & 1.011 & - & 0.978 & 0.999 & - & 0.977 & 0.992 & - \\
\hline $\mathrm{C}_{1}=\mathrm{O}_{1}$ & 1.234 & 1.257 & - & 1.232 & 1.256 & - & 1.235 & 1.259 & - \\
\hline $\mathrm{H}_{1} \cdots \mathrm{O}_{1}$ & 1.946 & 1.734 & - & 1.984 & 1.817 & - & 1.985 & 1.841 & - \\
\hline $\mathrm{C}_{2}=\mathrm{O}_{2}$ & - & - & 1.271 & - & - & 1.262 & - & - & 1.261 \\
\hline $\mathrm{H}_{1}-\mathrm{O}_{1}$ & - & - & 0.979 & - & - & 0.980 & - & - & 0.987 \\
\hline $\mathrm{H}_{1} \cdots \mathrm{O}_{2}$ & - & - & 1.996 & - & - & 1.992 & - & - & 1.902 \\
\hline
\end{tabular}



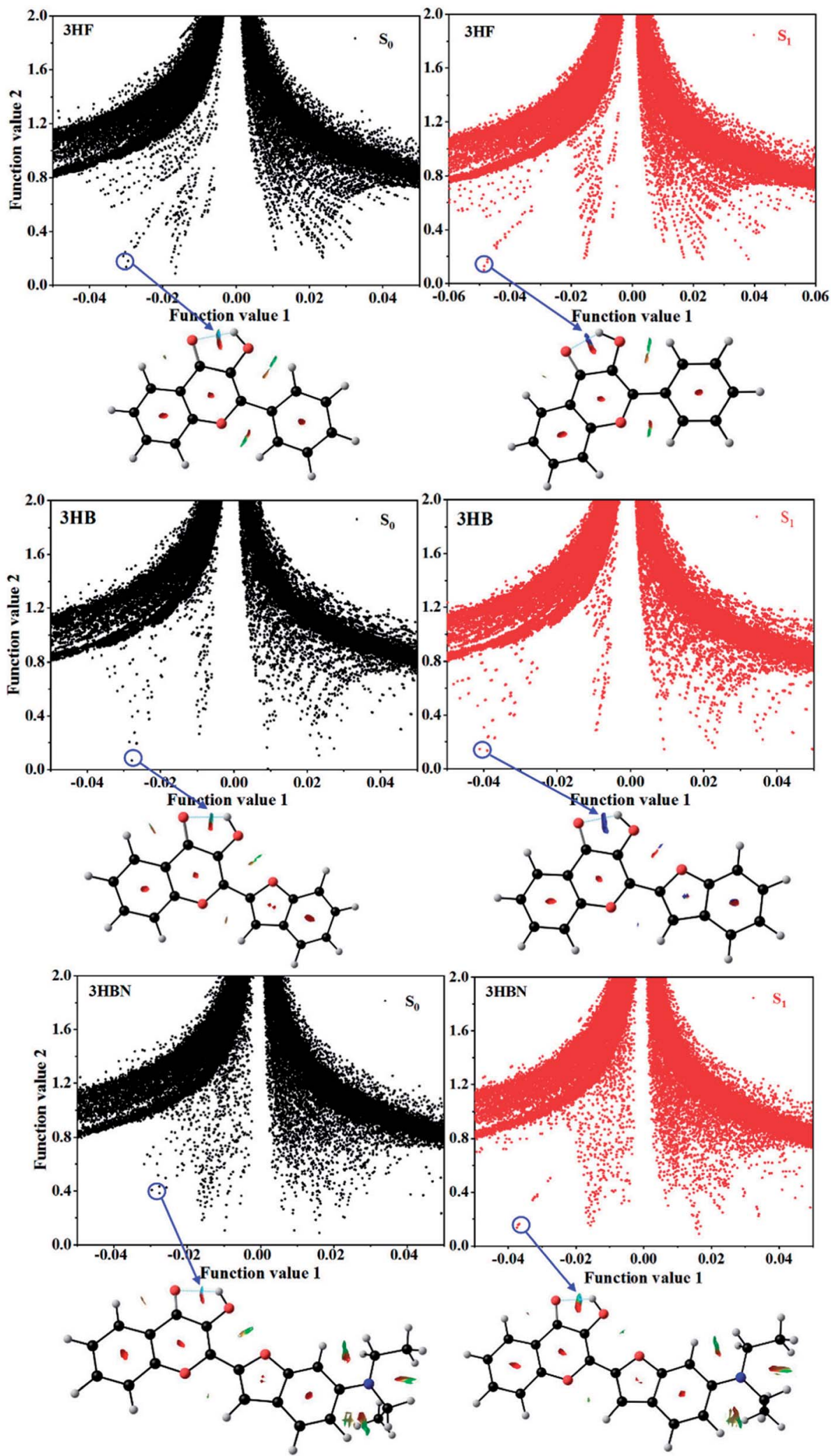

Fig. 2 Plots of function value 1 versus function value 2 for 3HF, 3HB and $3 \mathrm{HBN}$ conformations in the ground-state and excited-state, as well as the assignments of each spike by gradient isosurfaces.

dispersion-solvent-structure terms in Truhlar and coworkers' SMD solvation model for toluene was carried out. ${ }^{\mathbf{4 2 - 4 4}}$ The reduced density gradient (RDG) function was introduced to investigate non-covalent interactions using the Multiwfn program. ${ }^{4-47}$ Chemcraft software was exploited to visualize the RDG in a real place. ${ }^{48}$ Potential energy curves have been 
performed beginning from normal to tautomer form, constraining the bond strength $\mathrm{O}-\mathrm{H}$ and optimizing all other coordinates at every scan point. The transition state (TS) search was performed and verified by just only one imaginary frequency of energy second derivatives (Hessian) matrices. ${ }^{\mathbf{9}}$

\section{Results and discussion}

\subsection{Electronic excitation energies and optimized geometric structures}

Based on Salaeh et al. 's theoretical research on the ESIPT process of $3 \mathrm{HF}$ molecule under different polar solvents at the B3LYP/TZVP level, solvation effect and more functionals (PBE0, M062X, B3LYP and CAM-B3LYP) were selected to ensure the validity of the calculation for our studied system. ${ }^{19}$ The electronic excitation energies of different functional were calculated and corresponding results are shown in Table 1. The results indicate that the PBE0 functional keeps the most satisfactory agreement with experimental results. ${ }^{24}$ Therefore, the PBE0 functional was adopted in our whole calculation process. The oscillator strengths in first two excited-state and corresponding orbital transitions of $3 \mathrm{HF}, 3 \mathrm{HB}$ and $3 \mathrm{HBN}$ at PBE0/TZVP level are presented in Table 2 . The $\mathrm{S}_{0} \rightarrow \mathrm{S}_{1}$ transitions correspond to the maximum oscillator strength of $3 \mathrm{HF}, 3 \mathrm{HB}$, and $3 \mathrm{HBN}$ structures. On the contrary, the $\mathrm{S}_{0} \rightarrow \mathrm{S}_{2}$ transitions for these three structures have small oscillator strengths. Therefore, only the $S_{0} \rightarrow S_{1}$ transitions of $3 \mathrm{HF}, 3 \mathrm{HB}$ and $3 \mathrm{HBN}$ and their molecular orbitals are considered in current system.

The optimized lowest energy geometric configurations of $3 \mathrm{HF}, 3 \mathrm{HB}$ and $3 \mathrm{HBN}$ in the ground-state $\left(\mathrm{S}_{0}\right)$ and the first excited-state $\left(S_{1}\right)$ are shown in Fig. 1 . Some crucial bond length parameters for these three stable structures are listed in Table 3. For $3 \mathrm{HF}$, the bond lengths of $\mathrm{O}_{2}-\mathrm{H}_{1}$ and $\mathrm{C}_{1}=\mathrm{O}_{1}$ are increased from $0.979 \AA$ and $1.234 \AA$ in the $\mathrm{S}_{0}$ state to $1.011 \AA$ and $1.257 \AA$ in

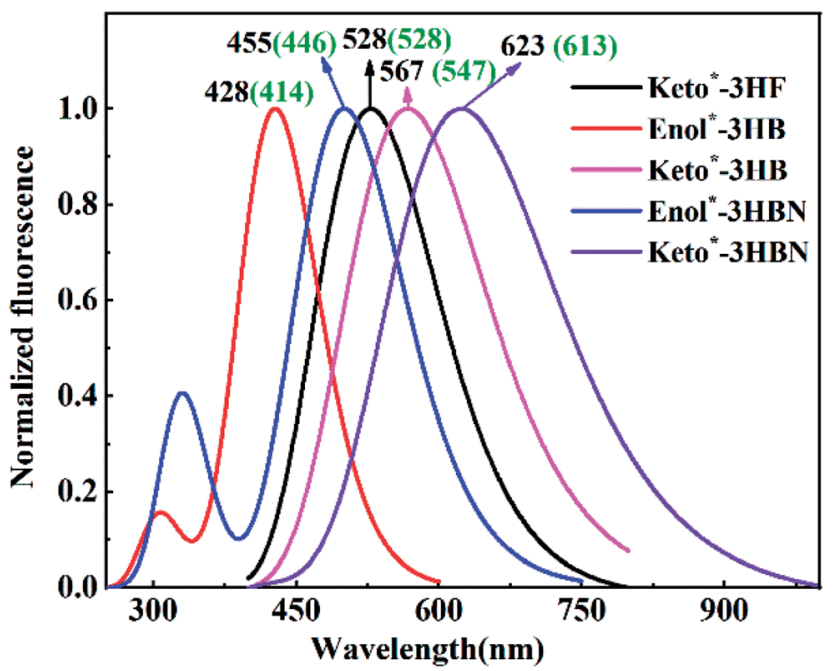

Fig. 3 Plotted normalized emission spectra of $3 \mathrm{HF}, 3 \mathrm{HB}$ and $3 \mathrm{HBN}$ in toluene solvent. Black wavelength values stand for theoretical simulation results and numbers in brackets represent experimental values (see ref. 24). the $\mathrm{S}_{1}$ state. The intramolecular hydrogen bond (IMHB) $\mathrm{O}_{2}-$ $\mathrm{H}_{1} \cdots \mathrm{O}_{1}$ length is decreased from $1.946 \AA$ in the $\mathrm{S}_{0}$ state to 1.734 $\AA$ in the $\mathrm{S}_{1}$ state. Moreover, for $3 \mathrm{HB}$ structure, the bond lengths of $\mathrm{O}_{2}-\mathrm{H}_{1}, \mathrm{C}_{1}=\mathrm{O}_{1}$ and $\mathrm{O}_{2}-\mathrm{H}_{1} \cdots \mathrm{O}_{1}$ change from $0.978 \AA$, $1.232 \AA$ and $1.984 \AA$ to $0.999 \AA, 1.256 \AA$ and $1.817 \AA$, respectively. For $3 \mathrm{HBN}$, the bond lengths of $\mathrm{O}_{2}-\mathrm{H}_{1}, \mathrm{C}_{1}=\mathrm{O}_{1}$ and $\mathrm{O}_{2}-\mathrm{H}_{1} \cdots \mathrm{O}_{1}$ from $0.977 \AA$ А $1.235 \AA$ and $1.985 \AA$ change to $0.992 \AA, 1.259 \AA$ and $1.841 \AA$. Based on the above analysis of bond length, the IMHB was significantly strengthened in the $S_{1}$ state compared with that in the $S_{0}$ state for these three stable structures. Furthermore, it should be noted that the intramolecular hydrogen bond lengths for these three structures in the $\mathrm{S}_{1}$ state were increased from 1.734 $\AA$ for $3 \mathrm{HF}, 1.817 \AA$ for $3 \mathrm{HB}$ and to $1.841 \AA$ for $3 \mathrm{HBN}$. This phenomenon indicates that the modifications of molecular structure decrease the strength of excited-state IMHBs.

\subsection{Non-covalent interactions analysis}

An approach developed by Yang et al. to visualize non-covalent interactions in real space could be adopted to investigate hydrogen bond properties. ${ }^{45,46}$ Different interaction types and corresponding intensities can be clearly displayed by electron density $(\rho(r))$ and their reduced density gradient (RDG) isosurfaces analysis. Herein, the eqn (1) of RDG function can be expressed as

$$
\operatorname{RDG}(r)=\frac{1}{2\left(3 \pi^{2}\right)^{1 / 3}} \frac{|\nabla \rho(r)|}{\rho(r)^{4 / 3}}
$$

Furthermore, based on Bader's Atoms in Molecules theory, ${ }^{50}$ the second largest eigenvalue $\lambda_{2}$ of Hessian matrix of electron density and $\rho(r)$ can be contacted in the eqn (2)

$$
\Omega(r)=\operatorname{sign}\left(\lambda_{2}(r)\right) \rho(r)
$$

Herein the eigenvalue $\lambda_{2}>0$ and $\lambda_{2}<0$ stand for bonding and anti-bonding interactions, respectively. The $\operatorname{sign}\left(\lambda_{2}(r)\right) \rho(r)$ is large and negative value stands for attractive interaction like hydrogen bond interaction. In contrast, $\operatorname{sign}\left(\lambda_{2}(r)\right) \rho(r)$ is large and positive value means the anti-bonding interaction like steric repulsion interaction. The $\operatorname{sign}\left(\lambda_{2}(r)\right) \rho(r)$, nearly zero value, means van der Waals interaction. Herein, scatter

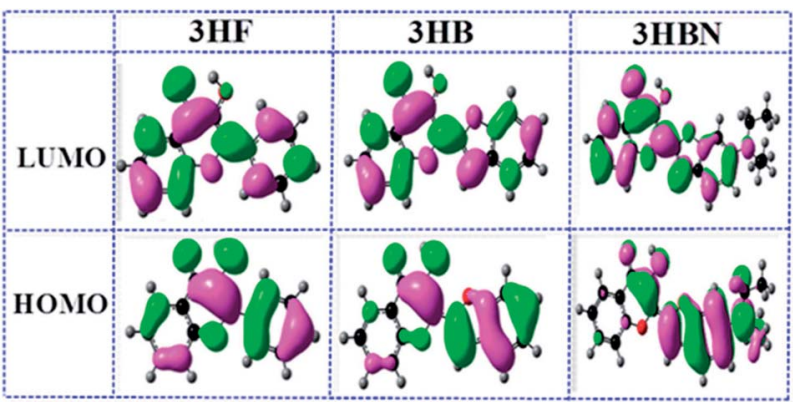

Fig. 4 Frontier molecular orbitals (HOMO and LUMO) of 3HF, 3HB and $3 \mathrm{HBN}$ structures. 
diagrams in $3 \mathrm{HF}, 3 \mathrm{HB}$, and $3 \mathrm{HBN}$ of the $\Omega(r)$ value (function value 1) versus the $\mathrm{RDG}(r)$ value (function value 2 ) are plotted in Fig. 2. The contour value is set as 0.5 . For $3 \mathrm{HF}$, the spike peaks almost located at -0.03 in $S_{0}$ state and -0.05 in $S_{1}$ state are observed and they have been confirmed to be the hydrogen bond interactions. Furthermore, the spike peak occurs left shift in the $S_{1}$ state compared with the $S_{0}$ state proved intramolecular hydrogen bond interactions are strengthened. For $3 \mathrm{HB}$ and $3 \mathrm{HBN}$ structures, the hydrogen bond interaction type and $\mathrm{S}_{1}$ state strengthen trend were further proved. By comparison, the strength of hydrogen bond interactions in the $\mathrm{S}_{0}$ state is similar since the spike peaks are close to -0.03 for these three structures. Compared with the spike peak of $3 \mathrm{HF}(-0.05)$ in the $\mathrm{S}_{1}$ state, the spike peaks of $3 \mathrm{HB}$ and $3 \mathrm{HBN}$ located in -0.04 and -0.037 , respectively. The spike peaks occur right-shift indicates the hydrogen bond interaction of $3 \mathrm{HF}$ was stronger than $3 \mathrm{HB}$ and 3 HBN structures. The results of non-covalent interactions greatly corroborate to the above-mentioned hydrogen-bonding length analysis results.

\subsection{Absorption and emission spectra analysis}

Klymchenko et al. have measured the absorption and the emission spectra of $3 \mathrm{HF}, 3 \mathrm{HB}$ and $3 \mathrm{HBN}$ using Cary 3 Bio Spectrophotometer and Quanta Master spectrofluorometer, respectively. ${ }^{24}$ The calculated absorption peaks of three target molecules at PBE0/TZVP level occur remarkable red-shift from $3 \mathrm{HF}(343 \mathrm{~nm})$ to $3 \mathrm{HB}(378 \mathrm{~nm})$ and to $3 \mathrm{HBN}$ (455 nm), achieving a leap from ultraviolet (UV) to visible (VIS) band (see Table 1). Such a significant red-shift can be explained by the orbital transition energy gaps. The calculated results show that the energy level of highest occupied molecular orbital (HOMO) is elevated from $2.19 \mathrm{eV}$ (3HF) to $2.34 \mathrm{eV}$ (3HB) due to the extension of furan heterocycle. The lowest unoccupied molecular orbital (LUMO) energy level is pulled down from $6.41 \mathrm{eV}(3 \mathrm{HF})$ to $5.36 \mathrm{eV}(3 \mathrm{HBN})$ for the introduction of electron-withdrawing group (dialkylamino). Therefore, a conclusion can be drawn that the rise of HOMO orbital energy level and the decrease of LUMO orbital energy level effectively reduce the energy level gap between them and induce the red shift of the spectra.

For exploring the effects of chromophore length and electrondonor ability on the positions of emission spectra, simulated normalized emission spectra of $3 \mathrm{HF}, 3 \mathrm{HB}$ and $3 \mathrm{HBN}$ structures in toluene solvent are shown in Fig. 3. Upon photoexcitation to the $\mathrm{S}_{1}$, only the $528 \mathrm{~nm}$ Keto* fluorescence emission peak is observed for $3 \mathrm{HF}$ in toluene solvent, which can reasonably be explained through a fast proton transfer channel between normal and tautomer forms. ${ }^{20}$ For excited-state $3 \mathrm{HB}$ and $3 \mathrm{HBN}$ structures, two fluorescence emission peaks are observed simultaneously. The Enol* form fluorescence peak is $428 \mathrm{~nm}$ for $3 \mathrm{HB}$ and $500 \mathrm{~nm}$ for $3 \mathrm{HBN}$, which is in accordance with experimental results (414 $\mathrm{nm}$ for $3 \mathrm{HB}$ and $508 \mathrm{~nm}$ for $3 \mathrm{HBN}$ ). Besides, the Keto* form fluorescence is $567 \mathrm{~nm}(3 \mathrm{HB})$, and $623 \mathrm{~nm}(3 \mathrm{HBN})$, which is also in agreement with the experiment peak values $547 \mathrm{~nm}$ and $613 \mathrm{~nm}$, respectively. Therefore, as the molecular structure changes from $3 \mathrm{HF}$ to $3 \mathrm{HB}$ and to $3 \mathrm{HBN}$, the emission spectra were greatly shifted to the red region.

\subsection{Frontier molecular orbitals analysis}

It is known to all that charge transfer deriving from photoexcitation could reasonably describe the nature of electronically excited-state dynamic behavior. ${ }^{51-53}$ In order to have a clear-cut description of charge distribution and charge transfer, the

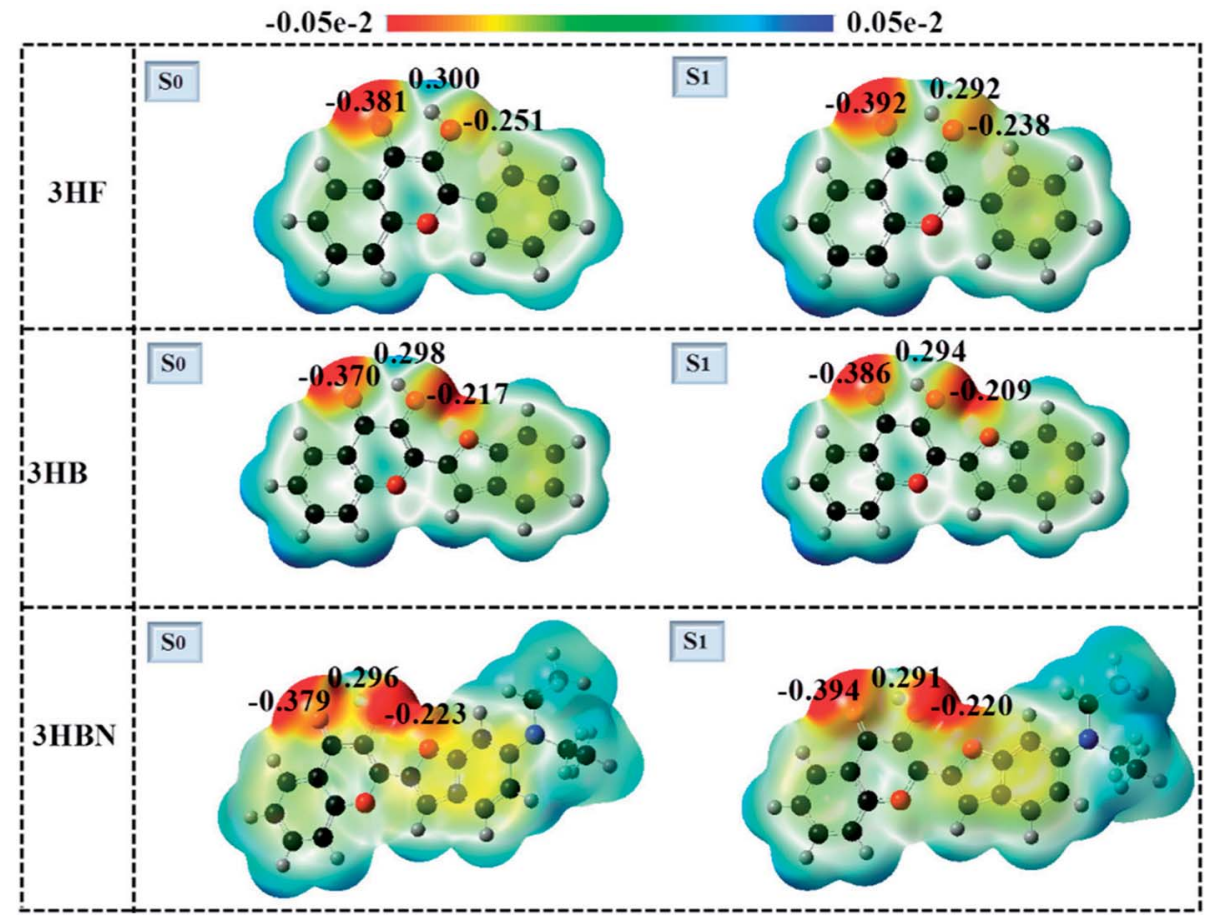

Fig. 5 The molecular electrostatic potentials of $3 \mathrm{HF}, 3 \mathrm{HB}$, and $3 \mathrm{HBN}$ in the ground-state and excited-state. 
HOMO and LUMO of frontier molecular orbitals (FMOs) of 3HF, $3 \mathrm{HB}$, and $3 \mathrm{HBN}$ structures are displayed in Fig. 4 . The $\pi$ character of HOMO and the $\pi^{*}$ character of LUMO can be seen clearly, which indicates $S_{0} \rightarrow S_{1}$ transition is belong to predominantly $\pi \pi^{*}$ type transition. For $3 \mathrm{HF}, 3 \mathrm{HB}$, and $3 \mathrm{HBN}$ structures, the charge transfer of significant sites involved in intramolecular hydrogen bond is analyzed. As seen in Fig. 4, conspicuous charge transfer phenomenon both in the HOMO and in the LUMO is observed from $3 \mathrm{HF}$ changes to $3 \mathrm{HB}$ and $3 \mathrm{HBN}$ whereas the charge transfer direction of two orbitals is opposite. Specifically, when the molecular structure changes from $3 \mathrm{HF}$ to $3 \mathrm{HBN}$ along with the charge in the HOMO is transferred from phenyl ring on the left to diethylamino site located on the right. It is interesting that the exact opposite transition happens in the LUMO. After molecular orbitals from HOMO transition to LUMO, the electron densities of hydroxyl moiety decrease and that of the acceptor $\mathrm{O}$ atom increase for these three structures. As a consequence, obvious intramolecular charge transfer leads to the $\mathrm{H}$ atom of hydroxyl moiety more acidic and the $\mathrm{O}$ atom of carboxyl group more basic. Therefore, the qualitative variation of the basicity/acidity is a strong proof for the occurrence of ICT process of target molecules.

\subsection{Electrostatic potential analysis}

A close inspection of the charge distribution over the atoms involved in intramolecular hydrogen-bond directly provides a window towards examining the ESIPT process. The electrostatic potential (ESP) pictures in the ground-state and excited-state of $3 \mathrm{HF}, 3 \mathrm{HB}$, and $3 \mathrm{HBN}$ are drawn in Fig. 5. The color changes from red, green to blue delegate the increasing of ESP, which is closely connected with the changes of electron density. The red and blue regions stand for negative ESP and positive ESP, respectively. For $3 \mathrm{HF}$, the negative charge distribution on $\mathrm{O}$ atom of $\mathrm{O}-\mathrm{H}$ moiety decreases from 0.251 in the $S_{0}$ state to 0.238 in the $S_{1}$ state, whereas the $\mathrm{O}$ atom of $\mathrm{C}=\mathrm{O}$ moiety increases from 0.381 to 0.392. Furthermore, similar charge change trend also was found both in $3 \mathrm{HB}$ and $3 \mathrm{HBN}$ structures. To be more specific, for $3 \mathrm{HB}$ and $3 \mathrm{HBN}$ structures, the charge density of donor $\mathrm{O}$ in the $\mathrm{O}-\mathrm{H}$ moiety was decreased from $0.217,0.223$ in the $S_{0}$ state to 0.209 , 0.220 in the $S_{1}$ state, respectively. Meanwhile, the charge density of acceptor $\mathrm{O}$ in $\mathrm{C}=\mathrm{O}$ moiety was increased from $0.370,0.379$ in the $\mathrm{S}_{0}$ state to $0.386,0.394$ in the $\mathrm{S}_{1}$ state, respectively. Therefore, the conclusion can be drawn based on above ESP analysis that the capabilities of electron-withdrawing group $(\mathrm{C}=\mathrm{O})$ and electron-donating group $(\mathrm{O}-\mathrm{H})$ were strengthened in the $\mathrm{S}_{1}$ state compared with those in the $S_{0}$ state for these three structures. Such strengthen effectively shortens excited-state intramolecular hydrogen bond distance and leads to the proton transfer processes are easier to proceed in the $S_{1}$ state than $S_{0}$ state.

\subsection{Infrared spectra analysis}

Infrared (IR) spectrum is an effective tool to analysis intra- and inter-molecular hydrogen bond strengthening or weakening through an electronic spectral red-shift or blue-shift. ${ }^{54,55}$ As shown in Fig. 6, the IR spectra of different electronic states of $3 \mathrm{HF}, 3 \mathrm{HB}$, and $3 \mathrm{HBN}$ structures are depicted. Obviously, two stretching vibrational peaks connected to intramolecular hydrogen bond were interested for $3 \mathrm{HF}, 3 \mathrm{HB}, 3 \mathrm{HBN}$ structures. For $3 \mathrm{HF}, \mathrm{C}_{1}=\mathrm{O}_{1}$ and $\mathrm{H}_{1}-\mathrm{O}_{2}$ stretching vibrational peaks change from $S_{0}$ state $1709 \mathrm{~cm}^{-1}$ to $S_{1}$ state $1663 \mathrm{~cm}^{-1}$ and from $S_{0}$ state $3567 \mathrm{~cm}^{-1}$ to $S_{1}$ state $3057 \mathrm{~cm}^{-1}$ respectively. Meanwhile, the similar red-shift trend also was found in $3 \mathrm{HB}$ and $3 \mathrm{HBN}$ structures. Compared with the $S_{0}$ state, the $51 \mathrm{~cm}^{-1}$ red-shift for $\mathrm{C}_{1}=\mathrm{O}_{1}$ and $351 \mathrm{~cm}^{-1}$ dramatic red-shift for $\mathrm{H}_{1}-\mathrm{O}_{2}$ were found in the $\mathrm{S}_{1}$ state for $3 \mathrm{HB}$. Similarly, the $44 \mathrm{~cm}^{-1}$ red-shift for $\mathrm{C}_{1}=$ $\mathrm{O}_{1}$ and $254 \mathrm{~cm}^{-1}$ red-shift for $\mathrm{H}_{1}-\mathrm{O}_{2}$ were found for $3 \mathrm{HBN}$. So conspicuous red-shift as a result of photoexcitation indicates that the intramolecular hydrogen bond in the excited-state was strengthened for these three structures, which further demonstrated above discussion regarding bond length and noncovalent interactions. After excited-state intramolecular proton transfer process, the tautomer species were formed, the new intramolecular hydrogen bonds replace original intramolecular

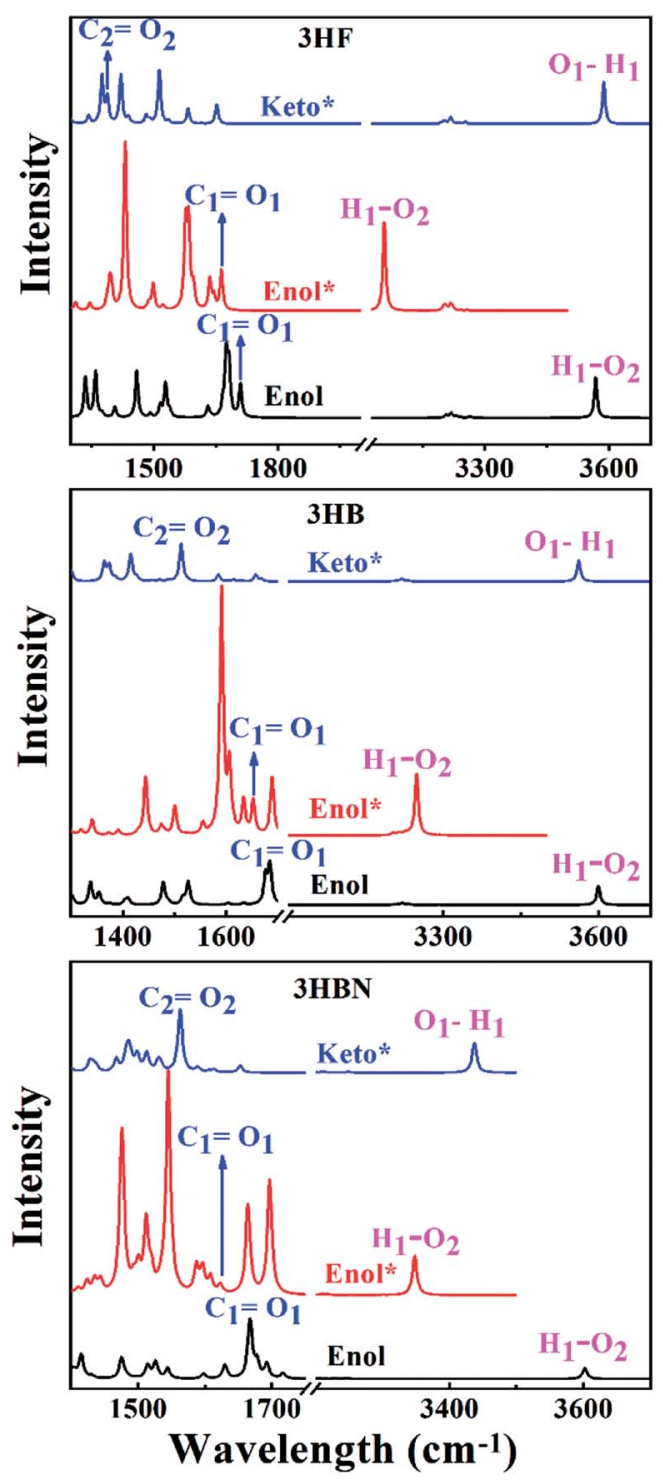

Fig. 6 Calculated IR spectra of the 3HF, 3HB, and 3HBN in different electronic states. Black line, red line and blue line stands for Enol, Enol* and Keto* form of IR spectra, respectively. 
hydrogen bonds. The new stretching vibrational peaks $\mathrm{C}_{2}=\mathrm{O}_{2}$, $\mathrm{O}_{1}-\mathrm{H}_{1}$ for $3 \mathrm{HF}, 3 \mathrm{HB}$ and $3 \mathrm{HBN}$ are explored. Therefore, it is reasonable to say that the analysis of IR spectra indirectly depicts the occurrence of ESIPT process of $3 \mathrm{HF}, 3 \mathrm{HB}$ and $3 \mathrm{HBN}$ structures. The detailed ESIPT process is analyzed in the following Potential energy curve section.

\subsection{Potential energy curves and mechanism analysis}

To gain more insight into the intramolecular proton transfer mechanism, the potential energy curves for $3 \mathrm{HF}, 3 \mathrm{HB}$, and 3 HBN structures in the $S_{0}$ and $S_{1}$ states are constructed and shown in Fig. 7. In the $\mathrm{S}_{0}$ state, the potential barrier is $12.38 \mathrm{kcal} \mathrm{mol}^{-1}$ for $3 \mathrm{HF}, 13.62 \mathrm{kcal} \mathrm{mol}^{-1}$ for $3 \mathrm{HB}$, and $13.49 \mathrm{kcal} \mathrm{mol}^{-1}$ for $3 \mathrm{HBN}$ respectively. Obviously, it is difficult

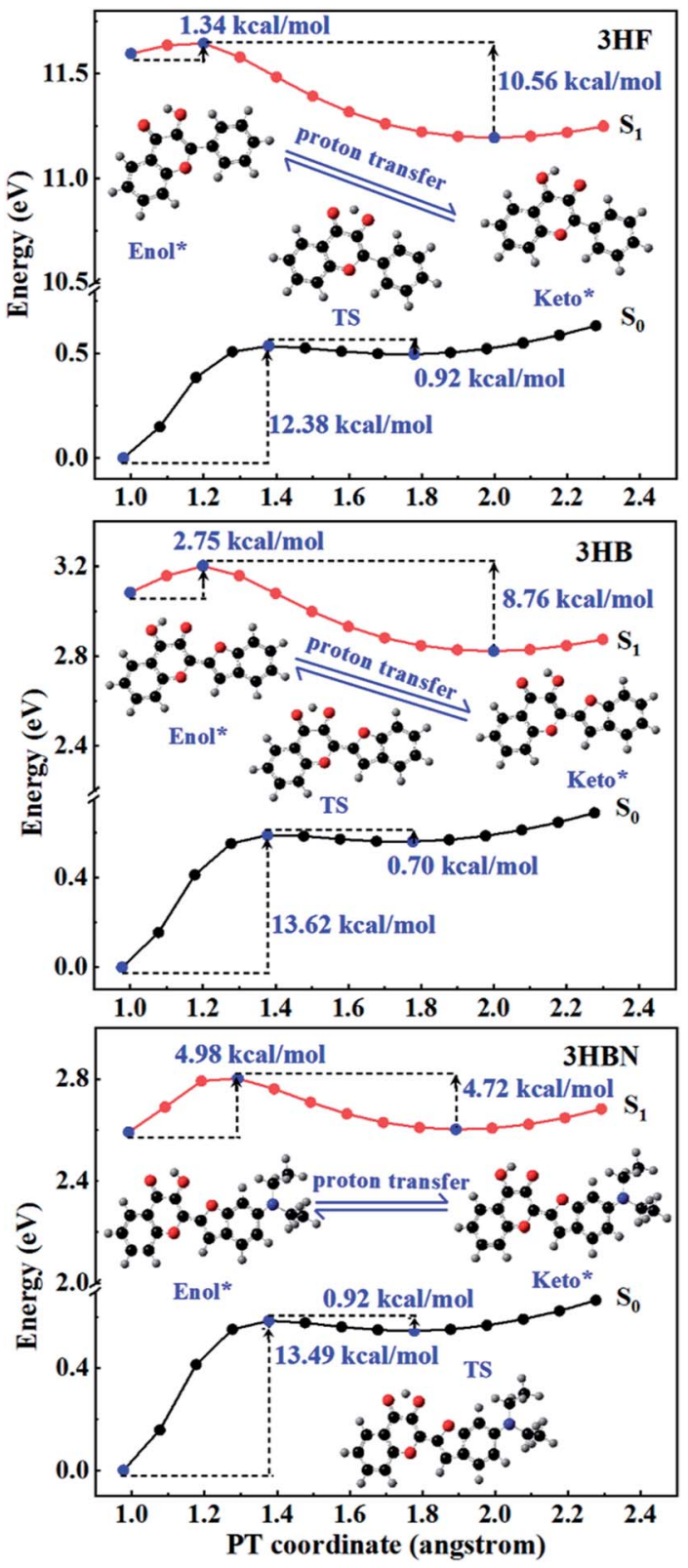

Fig. 7 The potential energy curves of the ground-state and excitedstate for $3 \mathrm{HF}, 3 \mathrm{HB}$, and $3 \mathrm{HBN}$ structures. to proceed intramolecular proton transfer spontaneously in the ground-state due to the higher energy barriers. After vertical excitation to $S_{1}$ state, the potential barrier is $1.34 \mathrm{kcal} \mathrm{mol}^{-1}$ for $3 \mathrm{HF}, 2.75 \mathrm{kcal} \mathrm{mol}^{-1}$ for $3 \mathrm{HB}$, and $4.98 \mathrm{kcal} \mathrm{mol}^{-1}$ for $3 \mathrm{HBN}$ respectively. Lower excited-state barriers demonstrate that trigger ESIPT process for $3 \mathrm{HF}, 3 \mathrm{HB}$ and $3 \mathrm{HBN}$ is relatively easy. Furthermore, for the reverse ESIPT process, the potential barriers decrease from $10.56 \mathrm{kcal} \mathrm{mol}^{-1}$ to $8.76 \mathrm{kcal} \mathrm{mol}^{-1}$ and to $4.72 \mathrm{kcal} \mathrm{mol}^{-1}$ with the structure changes from $3 \mathrm{HF}, 3 \mathrm{HB}$ and to $3 \mathrm{HBN}$, which indicates that $3 \mathrm{HBN}$ structure easier to occur reverse ESIPT process compared with $3 \mathrm{HF}$ and $3 \mathrm{HB}$ structures. Meanwhile, a phenomenon should be noticed that the potential energy barriers of forward ESIPT get higher along with the strengthened ICT from $3 \mathrm{HF}$ to $3 \mathrm{HB}$ and to $3 \mathrm{HBN}$. Therefore, ESIPT and ICT processes are competitive mechanism in current research system.

\section{Conclusion}

In summary, we theoretically investigated the characteristics of intramolecular hydrogen bonds and dynamic behaviors of excited-state intramolecular proton transfer and intramolecular charge transfer for $3 \mathrm{HF}, 3 \mathrm{HB}$, and $3 \mathrm{HBN}$ structures. The calculation results demonstrated that proper site furan heterocycle extension and diethylamino donor group substitution based on $3 \mathrm{HF}$ structure effectively modulated the absorption peak values from UV to visible band. Moreover, the fluorescence emission wavelength of $3 \mathrm{HB}$ and $3 \mathrm{HNB}$ structures exhibited strong red shift compared with the original $3 \mathrm{HF}$ structure. More importantly, as the structure changes from $3 \mathrm{HF}$ to $3 \mathrm{HB}$ and to $3 \mathrm{HBN}$, intramolecular charge transfer characteristics got increased accompanied with higher energy barriers of excited-state intramolecular proton transfer. This observation leads to a conclusion that excited-state intramolecular proton transfer and intramolecular charge transfer processes are competitive mechanism. Therefore, reasonable structure design achieved the control of excited-state dynamic behaviors in the current system. We envision that our work would provide paradigmatic guidance for designing and developing new fluorescent sensors and organic radiation scintillators.

\section{Conflicts of interest}

The authors declare no competing interests.

\section{Acknowledgements}

This work was supported by the National Natural Science Foundation of China (No. 11574115); the Natural Science Foundation of Jilin Province of China (Grant No. 20150101063JC).

\section{References}

1 S. A. Ahmed, B. Maity, S. S. Duley and D. Seth, J. Photochem. Photobiol., B, 2017, 168, 132-141. 
2 A. Sytnik and I. Litvinyuk, Proc. Natl. Acad. Sci. U. S. A., 1996, 93, 12959-12963.

3 J. R. Dharia, K. F. Johnson and J. B. Schlenoff, Macromolecules, 1994, 27, 5167-5172.

4 S. M. Dennison, J. Guharay and P. K. Sengupta, Spectrochim. Acta, Part A, 1999, 55, 1127-1132.

5 O. P. Bondar, V. G. Pivovarenko and E. S. Rowe, Biochim. Biophys. Acta, 1998, 1368, 119-130.

6 E. Karakus, M. Ucuncu and M. Emrullahoglu, Anal. Chem., 2016, 88, 1039-1043.

7 G. J. Zhao and K. L. Han, J. Comput. Chem., 2008, 29, 20102017.

8 L. G. Korkina and I. B. Afanas'ev, Adv. Pharmacol., 1997, 38, 151-163.

9 A. Bocco, M.-E. Cuvelier, H. Richard and C. Berset, J. Agric. Food Chem., 1998, 46, 2123-2129.

10 R. Bouhamidi, V. Prevost and A. Nouvelot, Life Sci., 1998, 321, 31-38.

11 J. S. Chen, P. W. Zhou, L. Zhao and T. S. Chu, RSC Adv., 2014, 4, 254-259.

12 J. S. Chen, P. W. Zhou, S. Q. Yang, A. P. Fu and T. S. Chu, Phys. Chem. Chem. Phys., 2013, 15, 16183-16189.

13 Y. Li, J. S. Chen and T. S. Chu, J. Lumin., 2016, 179, 203-210. 14 J. S. Chen, L. Zhao and T. S. Chu, Journal of Atomic and Molecular Sciences, 2015, 6, 1-10.

15 A. S. Klymchenko, T. Ozturk and A. P. Demchenko, Tetrahedron Lett., 2002, 43, 7079-7082.

16 A. S. Klymchenko and A. P. Demchenko, Phys. Chem. Chem. Phys., 2003, 5, 461-468.

17 T. C. Swinney and D. F. Kelley, J. Chem. Phys., 1993, 99, 211221.

18 A. J. G. Strandjord and P. F. Barbara, J. Phys. Chem., 1985, 89, 2355-2361.

19 R. Salaeh, C. Prommin, W. Chansen, K. kerdpol, R. Daengngern and N. Kungwan, J. Mol. Liq., 2018, 252, 428-438.

20 P.-T. Chou, M. L. Martinez and J. H. Clements, J. Phys. Chem., 1993, 97, 2618-2622.

21 A. P. Demchenko, K. C. Tang and P. T. Chou, Chem. Soc. Rev., 2013, 42, 1379-1408.

22 A. S. Klymchenko, V. G. Pivovarenko, T. Ozturk and A. P. Demchenko, New J. Chem., 2003, 27, 1336-1343.

23 A. P. Demchenko, S. Ercelen, A. D. Roshal and A. S. Klymchenko, Pol. J. Chem., 2002, 76, 1287-1299.

24 A. S. Klymchenko, T. Ozturk, V. G. Pivovarenkob and A. P. Demchenko, Tetrahedron Lett., 2001, 42, 7967-7970.

25 A. S. Klymchenko, T. Ozturk, V. G. Pivovarenko and A. P. Demchenko, Can. J. Chem., 2001, 79, 358-363.

26 M. J. Frisch, G. W. Trucks, H. B. Schlegel, G. E. Scuseria, M. A. Robb, J. R. Cheeseman, G. Scalmani, V. Barone, B. Mennucci, G. A. Petersson, H. Nakatsuji, M. Caricato, X. Li, H. P. Hratchian, A. F. Izmaylov, J. Bloino, G. Zheng, J. L. Sonnenberg, M. Hada, M. Ehara, K. Toyota, R. Fukuda, J. Hasegawa, M. Ishida, T. Nakajima, Y. Honda, O. Kitao, H. Nakai, T. Vreven, J. A. Montgomery Jr, J. E. Peralta, F. Ogliaro, M. Bearpark, J. J. Heyd, E. Brothers, K. N. Kudin, V. N. Staroverov, T. Keith,
R. Kobayashi, J. Normand, K. Raghavachari, A. Rendell, J. C. Burant, S. S. Iyengar, J. Tomasi, M. Cossi, N. Rega, J. M. Millam, M. Klene, J. E. Knox, J. B. Cross, V. Bakken, C. Adamo, J. Jaramillo, R. Gomperts, R. E. Stratmann, O. Yazyev, A. J. Austin, R. Cammi, C. Pomelli, J. W. Ochterski, R. L. Martin, K. Morokuma, V. G. Zakrzewski, G. A. Voth, P. Salvador, J. J. Dannenberg, S. Dapprich, A. D. Daniels, O. Farkas, J. B. Foresman, J. V. Ortiz, J. Cioslowski and D. J. Fox, Gaussian 09, revision B 01, Gaussian, Inc., Wallingford, CT, 2010.

27 C. Adamo and V. Barone, Theor. Chem. Acc., 2000, 105, 169172.

28 M. Ernzerhof and G. E. Scuseria, J. Chem. Phys., 1999, 110, 5029-5036.

29 J. P. Perdew, K. Burke and M. Ernzerhof, Phys. Rev. Lett., 1996, 77, 3865-3868.

30 J. P. Perdew, M. Ernzerhof and K. Burke, J. Chem. Phys., 1996, 105, 9982-9985.

31 A. Schäfer, C. Huber and R. Ahlrichs, J. Chem. Phys., 1994, 100, 5829-5835.

32 F. Furche and R. Ahlrichs, J. Chem. Phys., 2002, 117, 74337447.

33 A. D. Becke, J. Chem. Phys., 1993, 98, 5648-5652.

34 C. Z. Li, Y. G. Yang, C. Ma and Y. F. Liu, RSC Adv., 2016, 6, 5134-5140.

35 D. P. Yang, J. F. Zhao, M. Jia and X. Y. Song, RSC Adv., 2017, 7, 34034-34040.

36 D. P. Yang, G. Yang, J. F. Zhao, R. Zheng and Y. S. Wang, RSC Adv., 2017, 7, 1299-1304.

37 H. P. Ma and J.-D. Huang, RSC Adv., 2016, 6, 96147-96153.

38 J. F. Zhao, J. S. Chen, Y. L. Cui, J. Wang, L. X. Xia, Y. M. Dai, P. Song and F. C. Ma, Phys. Chem. Chem. Phys., 2015, 17, 1142-1150.

39 D. P. Yang, Y. G. Yang and Y. F. Liu, J. Cluster Sci., 2014, 25, 467-481.

40 G.-Y. Li and T. S. Chu, Phys. Chem. Chem. Phys., 2011, 13, 20766-20771.

41 P. W. Zhou and K. L. Han, Acc. Chem. Res., 2018, 51, 16811690.

42 C. Z. Li, C. Ma, D. L. Li and Y. F. Liu, J. Lumin., 2016, 172, 2933.

43 G. Scalmani, M. J. Frisch, B. Mennucci, J. Tomasi, R. Cammi and V. Barone, J. Chem. Phys., 2006, 124, 094107.

44 A. V. Marenich, C. J. Cramer and D. G. Truhlar, J. Phys. Chem. B, 2009, 113, 6378-6396.

45 J. Contreras-Garcia, E. R. Johnson, S. Keinan, R. Chaudret, J.-P. Piquemal, D. N. Beratan and W. T. Yang, J. Chem. Theory Comput., 2011, 7, 625-632.

46 E. R. Johnson, S. Keinan, P. Mori-Sanchez, J. ContrerasCarcia, A. J. Cohen and W. T. Yang, J. Am. Chem. Soc., 2010, 132, 6498-6506.

47 T. Lu and F. W. Chen, J. Comput. Chem., 2012, 33, 580-592. 48 Website of ChemCraft software, https:// www.chemcraftprog.com.

49 A. E. Shchavlev, A. N. Pankratov and A. V. Shalabay, J. Phys. Chem. A, 2005, 109, 4137-4148. 
50 W. Tang, E. Sanville and G. Henkelman, J. Phys.: Condens. Matter, 2009, 21, 084204.

51 J. S. Chen, R. Z. Liu, Y. Yang and T. S. Chu, Theor. Chem. Acc., 2014, 133, 1411.

52 J. F. Zhao and P. Li, RSC Adv., 2015, 5, 73619-73625.
53 P. Song, Y. Z. Li, F. C. Ma, T. Pullerits and M. T. Sun, J. Phys. Chem. C, 2013, 117, 15879-15889.

54 J. S. Chen, M. H. Yuan, J. P. Wang, Y. Yang and T. S. Chu, J. Phys. Chem. A, 2014, 118, 8986-8995.

55 J. S. Chen, P. W. Zhou, G. Y. Li, T. S. Chu and G. Z. He, J. Phys. Chem. B, 2013, 117, 5212-5221. 